\title{
ANÁLISIS DE LOS ÍNDICE H, G Y R EN EL SECTOR AGROPECUARIO CUBANO A TRAVÉS DE SCOPUS, 2005-2009
}

\author{
Ibis Anette Lozano Díaz*
}

Grupo de Estudios Métricos. Biblioteca Nacional de Ciencia y Técnica de Cuba (BNCT). Instituto de Información Científica y Tecnológica de Cuba (IDICT).

\section{Yaniris Rodríguez Sánchez **}

Grupo de Estudios Métricos. Biblioteca Nacional de Ciencia y Técnica de Cuba (BNCT). Instituto de Información Científica y Tecnológica de Cuba (IDICT).

Resumen: La agricultura ocupa un reglón de importancia dentro de la economía cubana, por tal razón los estudios en este sector de la ciencia se han incrementado. Esta investigación tiene como objetivo principal analizar las ciencias agropecuarias cubanas a partir de la producción científica, impacto y rendimiento de los autores cubanos dedicados a la investigación del sector agropecuario, durante el período 2005-2009 en la base de datos SCOPUS. Se determinan los índices de productividad atendiendo a variables como: año, autor y revista. Se analiza el impacto de los resultados científicos a partir del total de citas recibidas, el total de artículos citados y el promedio de citas por artículo. Se calcula el índice H, G, A y R para 42 autores más productivos y los autores que reciben más de cuatro citas durante el período de estudios, con el propósito de comparar ambas muestras y determinar si los investigadores más productivos coinciden con los más citados. Se determinó la correlación los indicadores calculados, así como las distribuciones estadísticas. Se comprueba que los investigadores más productivos coinciden con los más citados y, por tanto, presentan índices $\mathrm{H}, \mathrm{G}, \mathrm{A}$ y $\mathrm{R}$ más favorables. Se considera una alternativa posible la incorporación de los análisis de citas, así como de los indicadores derivados de éstos, a los procesos de evaluación y análisis de la actividad científica para valorar los avances en el sector agropecuario.

Palabras clave: Indicadores bibliométricos; análisis de citas; índice $\mathrm{H}$; índice G; índice R; autores líderes; agricultura.

Title: ANALYSIS OF THE INDEX $\mathrm{H}, \mathrm{G}$ AND $\mathrm{R}$ IN THE CUBAN AGRICULTURAL SECTOR THROUGH SCOPUS, 2005-2009.

Abstract: Agriculture has a screed of importance in the Cuban economy, for this reason the studies in this field of science have increased. The principal research aims to analyze the Cuban agricultural sciences from the scientific output, impact and performance of Cuban authors dedicated to research in the agricultural sector during the period 2005-2009 in the SCOPUS database. Determining productivity rates in response to variables such as age, author and journal. It is analyzed the impact of

\footnotetext{
*ibis@idict.cu, ibis.alozano@gmail.com

** yaniris@idict.cu, yrs201181@gmail.com
}

Recibido: 16/11/2010; 2ª revisión: 15/02/2011; aceptado: 05/09/2011.

LOZANO DÍAZ, I.A. y RODRÍGUEZ SÁNCHEZ, Y. Análisis de los índices H, G y R en el sector agropecuario cubano a través de Scopus, 2005-2009. Anales de Documentación, 2012, vol. 15, $\mathrm{n}^{\text {o }} 1$. http://dx.doi.org/10.6018/analesdoc.15.1.147641. 
scientific results from the total citations, total cited articles and the average citations per paper. H, G, A and R Index is calculated for the 42 most productive authors and all the authors who received more than four cites during the study period, in order to compare the two samples and determine whether the most productive researchers agree with the most cited. The correlation is calculated indicators and statistical distributions. It is shown that the most productive researchers agree with the most cited and, therefore, have H, G, A and R indexes are more favorable. It is considered a possible alternative incorporating citation analysis and indicators derived from them, the processes of assessment and analysis of scientific activity to assess progress in the agricultural sector.

Keywords: Bibliometrics; citation analysis; $\mathrm{H}$ index; index G; R index; lead authors; agriculture.

\section{INTRODUCCIÓN}

La evaluación de conocimientos resultantes de la investigación científica constituye un componente indispensable para los sistemas de I+D+I en cualquier país; de ahí la necesidad de establecer parámetros que faciliten validar la calidad de la investigación. Las Ciencias de la información y en particular las disciplinas métricas tales como Bibliometría, Cienciometría e Informetría aportan herramientas de análisis que permiten reflejar el comportamiento de un área disciplinaria.

Establecer parámetros para validar la ciencia no es tarea fácil y a la vez constituye un objetivo primordial cuando se trata de evaluar la investigación científica. Existen instituciones que, adoptaron el factor de impacto de las revistas como un criterio más, para validar la calidad de la investigación y así reconocer el desempeño científico de aquellos investigadores líderes en una temática, parámetro que puede ser inadecuado, si se pretende hacer valoraciones de forma rigurosa y específica en una población de científicos con alta productividad. En este sentido, la Agencia Nacional de Evaluación de la Calidad y Acreditación (ANECA), y la Comisión Nacional Evaluadora de la Actividad Investigadora (CNEAI) en España, las cuales en un momento determinado consideraron como indicador de calidad y criterio de valoración de los investigadores individuales, la investigación publicada en revistas recogidas en los Journal Citation Reports (JCR) del ISI (Ruiz-Pérez, Delgado, Jiménez-Contreras, 2010, p. 899).

Evaluar el desempeño científico de los investigadores a partir del factor de impacto trae consigo graves consecuencias, pues en muchos casos los investigadores en ciencias sociales pueden ser desplazados por los investigadores especializados en ciencias como la física y las matemáticas. Esto se debe a la dependencia entre el número de citas bibliográficas de los trabajos y el tema y, a la falta de homogeneidad temática en los listados del Journal Citation Reports. El factor de impacto se creó para evaluar las revistas y no se diseñó para evaluar a investigadores de forma individual.

En este contexto aparece en el año 2005 el Índice H (Hirsch, 2005, p. 16569), creado por profesor de Física de la Universidad de California, Jorge E. Hirsch, más tarde, el Índice G (Egghe, 2006, p. 133), propuesto por Leo Egghe en el año 2006 y los Índices R y 
A (Jin; Liang; Rousseau y Egghe, 2007, p. 857), propuesto Jin BiHui y colaboradores en el 2007. Estos índices están encaminados a certificar el esfuerzo del desempeño científico durante la vida activa de un investigador dentro de la comunidad científica internacional, identificar los científicos más destacados en un campo del saber. Específicamente el índice $\mathrm{H}$, se ha utilizado de manera informal para clasificar aspirantes a puestos de investigador en centros de investigación (Ball, 2007, p. 4) (Bornmann y Hans-Dieter, 2009, p.4).

El índice H no está exento de limitaciones (Braun; Glänzel y Schubert, 2006, p. 170) (Costas y Bordons, 2007, p. 194) (Bornman; Mutz y Hans-Dieter, 2009, p. 1287), entre ellas se encuentra: la imposibilidad de comparar científicos de diferentes campos temáticos; no toma en cuenta la citas recibidas por el núcleo $\mathrm{H}$ de artículos, por lo que, han surgido alternativas como: el Índice G, Índice R, Índice A. Cuando se pretende medir carrera profesional existe desventaja entre los científicos consagrados y los que comienzan; refleja poco los cambios en el total de citas de los artículos más citados de un autor; es sensible a las autocitas. En cualquier caso, el índice $\mathrm{H}$ constituye un indicador que combina aspectos cuantitativos y cualitativos (cantidad de publicaciones y las citas) para evaluar la investigación de manera versátil a diferentes niveles de agregación y valorar la vida académica de un investigador.

\section{ANTECEDENTES}

El surgimiento de la metodología para calcular el Índice H, así como también obtener los Índices $G$ y $R$, facilitó a disímiles autores en el plano internacional (Scimago, 2006, p. 305), la realización de estudios de caso para comparar la actividad científica de investigadores de una disciplina, para la evaluación de publicaciones científicas y en evaluaciones a nivel institucional. Específicamente en Cuba, se ha determinado el núcleo de autores líderes para el área de las ciencias biomédicas, ciencias exactas y naturales, y recientemente, para el dominio de la comunicación y las ciencias de la información (Arenciabia-Jorge; Barrios-Almaguer; Fernández-Hernández y Carvajal-Espino, 2008, p. 156); (Arenciabia-Jorge y Carvajal-Espino, 2008, p. 4); (Dorta-Contreras; ArenciabiaJorge; Martí-Lahera y Araujo-Ruiz, 2008, p. 357) pero todavía no existe un estudio que muestre los investigadores más visibles en el sector agropecuario cubano a partir del cálculo de los índices H, G, A y R.

Identificar la productividad científica y la visibilidad de la ciencia cubana en las principales bases de datos internacionales ha constituido la línea de investigación más importante del "Sistema Nacional de Indicadores Cienciométricos para Cuba”, Proyecto Nacional de Ciencia y Tecnología del Instituto de información Científica y Tecnológica de Cuba, el cual ha estudiado constantemente la producción científica de todos los centros y universidades del país en temáticas como: la biomedicina, ciencias sociales y el sector agropecuario. Las bases de datos más utilizadas para estos estudios son el WoS y Scopus, los cuales son los más importantes índices de citas de revistas científicas y académicas a nivel internacional. 
El objetivo de la presente investigación es, estudiar las relaciones y la importancia que tienen los índices H, G, R y A en combinación con otros indicadores bibliométricos en la evaluación científica del sector agropecuario cubano, a partir de revistas indizadas en SCOPUS durante el período 2005-2009.

Se determinó seleccionar esta batería de indicadores para evaluar el comportamiento científico del sector agropecuario cubano, al tener en consideración que, de manera general el índice $\mathrm{H}$ es matemáticamente fácil de calcular, puede ser utilizado a diferentes niveles de agregación, tiende a valorar un esfuerzo científico desarrollado a lo largo de toda la vida académica de un autor, se puede emplear para evaluar determinados períodos de tiempo. Es un indicador robusto. No necesariamente el aumento o detrimento de la producción científica tiene efecto con el índice $\mathrm{H}$, aunque se ha demostrado que existe correlación entre ambos indicadores (Alonso; Cabrerizo; Herrera-Viedma y Herrera, 2010 p. 393).

En este sentido, los índices G, R y A para su cálculo tienen en cuenta la cantidad de citas recibidas por los artículos que constituyen el núcleo $\mathrm{H}$, es decir, del volumen de artículos más citados que se toman en consideración para el cálculo del Índice $\mathrm{H}$; elemento que no es relevante para el índice H (Egghe, 2006, p. 135) (Jin; Liang; Rousseau y Egghe, 2009, p. 240). Este aspecto permite obtener el número total de artículos en el núcleo $\mathrm{H}$ y también describe la producción científica núcleo de los científicos en una determinada rama de la ciencia.

El presente estudio se hace necesario en primer lugar porque: para cualquier rama de la ciencia, la actualización y evaluación sobre los temas de mayor relevancia que se discuten en sus diferentes sectores, constituyen aspectos que deben ser conocidos, así como, de los autores con mayor protagonismo y eficacia en el gremio científico. En segundo lugar, es preciso conocer un procedimiento bibliométrico que facilite identificar los mismos en el gran volumen de información que soporta una base de datos, así como tener determinado una batería de indicadores que permitan conocer el desempeño científico de un autor dentro de la comunidad científica nacional e internacional.

\section{MÉTODOS}

Se utilizó como fuente de información para este estudio la base de datos SCOPUS, creada por Elsevier. Se seleccionó esta base de datos porque constituye una base de datos de amplia cobertura citacional, lo que permite determinar la cantidad de citas que recibe cada artículo.

La estrategia de búsqueda que se empleó consistió en la identificación de la palabra "Cuba" en el campo Affiliation, limitando la búsqueda a las categorías temáticas: (Agriculture), (Agronomy), (Veterinary), (Environmental) en Scopus, y además se 
determinó como período de tiempo para el estudio los años 2005-2009. Se recuperó un total de 929 artículos.

Los artículos recuperados se exportaron directamente a una base de datos creada con el programa gestor de referencias bibliográficas EndNote X2, para normalizar el campo Autor, posteriormente se identificaron un total de 3282 autores, de los cuales 1422 recibieron más de una citas. Se estandarizó el nombre y los apellidos del total de autores. Luego se calculó la batería de indicadores que a continuación se presenta. Se determinó que solo se representan los autores con una producción mayor a siete artículos.

\section{Indicadores de Productividad}

- $\quad$ Número de artículos por: Año, Revista

\section{Indicadores calculados para cada autor}

- Número total de artículos publicados (Productividad)

- Número total de artículos citados (TACitados)

- Número total de citas recibidas (TcitasR)

- Total de citas en el núcleo H (TCnH)

- Promedio de citas recibidas por artículo (Prom de CXA)

- Índice H, de un investigador se determinó el mayor número de orden en el ranking con un total de citas recibidas igual o superior a ese número de orden (Hirsch, 2005, p. 16569)

- Índice G, de un investigador se identificó como el mayor número de orden en el ranking donde la sumatoria de citas recibidas por el autor fuera mayor o igual al cuadrado del número de orden (Egghe, 2006, p. 133)

- Índice R se calculó hallando la raíz cuadrada de la sumatoria de citas recibidas por el núcleo H de artículos más citados (Jin, Liang, Rousseau y Egghe, 2009, p. 236)

- Índice A se calculó dividiendo el total de citas en el núcleo H(TCnH) entre Índice H (Jin, Liang, Rousseau y Egghe, 2009, p. 237)

Se determinó el grado de asociación entre los indicadores estudiados a partir del coeficiente de correlación de Pearson con el propósito de comprobar si existe fuerte correlación estos índices específicamente en el campo de las ciencias agropecuarias cubanas. Las distribuciones estadísticas de cada indicador se determinaron mediante el programa Statistica 7.0 con el objetivo de obtener el valor promedio o valor representativo de los índices H, G, A y R en la muestra de 42 autores más productivos del sector agropecuario cubano.

\section{RESULTADOS}

Durante el período 2005-2009 se publicaron un total de 929 artículos científicos cubanos en la base de datos SCOPUS, de ellos 346 (37\%) recibieron un total de 1245 citas. El año 2006 con un total de 218 artículos constituye el año con mayor producción durante el período de estudio, y se muestra un comportamiento estable en la actividad 
científica, con tendencia a la disminución como lo demuestra el coeficiente de determinación $\mathrm{R}^{2}$. Además, se puede mencionar que la producción científica por año no está correlacionada con la producción científica total, si se considera que, existe inconstancia en la cantidad de artículos publicados por cada año. (Figura 1).

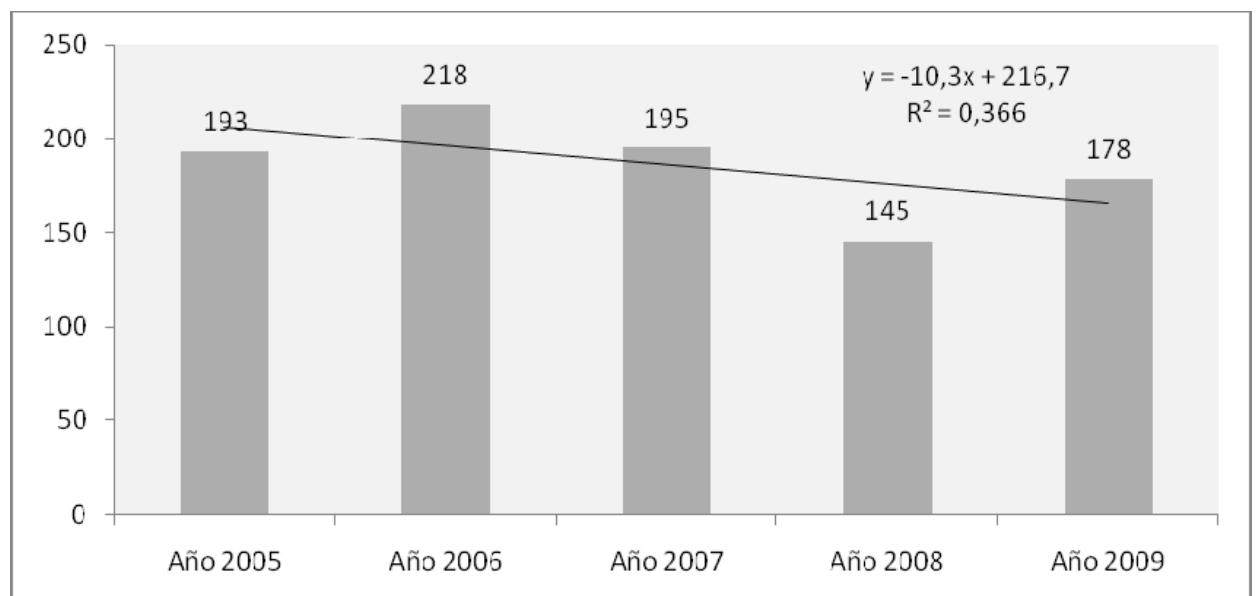

Figura 1. Producción científica por años en el campo de las ciencias agropecuarias a través de SCOPUS.

Importante señalar que solo 1,3\% del total de autores publicó 7 o más trabajos, y el $76 \%$ de investigadores publicaron un solo artículo; lo que demuestra que el campo de la ciencias agropecuarias cubanas en SCOPUS está representada por grupo pequeño de autores, y una gran cantidad de autores publican sus resultados científicos ocasionalmente.

El análisis de la base de datos SCOPUS permitió identificar un total de 308 revistas científicas, las cuales publicaron 929 artículos durante el período 2005-2009. En la Tabla I se representan aquellas revistas con más de 8 artículos publicados. La visibilidad de las ciencias agropecuarias cubanas no solo está representada por revistas extranjeras sino que el protagonismo en la muestra lo tiene la revista Cuban Journal of Agricultural Science. Además se corroboró que el 50\% de las publicaciones científicas que hacen visible la ciencia agropecuaria cubana pertenecen al cuartil 1. Por lo que se puede afirmar que los autores cubanos que estudian dicha temática publican en revistas con altos valores del Scimago Journal Rank (SJR). 


\begin{tabular}{|c|c|c|c|}
\hline Revistas & País & Artículos & $\begin{array}{l}\% \text { de artículos del } \\
\text { total de la muestra }\end{array}$ \\
\hline Cuban Journal of Agricultural Science & Cuba & 49 & 5,27 \\
\hline Vaccine & Holanda & 47 & 5,06 \\
\hline Plant Pathology & Reino Unido & 23 & 2,48 \\
\hline Journal of Essential Oil Research & EE.UU & 22 & 2,37 \\
\hline Mycotaxon & EE.UU & 20 & 2,15 \\
\hline $\begin{array}{l}\text { Zootaxa } \\
\text { Journal of Agricultural and Food } \\
\text { Chemistry }\end{array}$ & $\begin{array}{l}\text { Nueva } \\
\text { Zelanda } \\
\text { EE.UU }\end{array}$ & $\begin{array}{l}16 \\
12\end{array}$ & $\begin{array}{l}1,72 \\
1,29\end{array}$ \\
\hline Plant Cell, Tissue and Organ Culture & Holanda & 10 & 1,08 \\
\hline Journal of Medicinal Food & EE.UU & 10 & 1,08 \\
\hline Food Chemistry & Holanda & 8 & 0,86 \\
\hline Tuberculosis & EE.UU & 8 & 0,86 \\
\hline $\begin{array}{l}\text { Aquaculture } \\
\text { Livestock Research for Rural } \\
\text { Development }\end{array}$ & $\begin{array}{l}\text { Holanda } \\
\text { Colombia }\end{array}$ & 8 & 0,86 \\
\hline Ozone: Science and Engineering & Reino Unido & 8 & 0,86 \\
\hline Sugar Tech & India & 8 & 0,86 \\
\hline
\end{tabular}

Tabla I. Publicaciones científicas que hacen visible las ciencias agropecuarias cubanas a través de SCOPUS durante el período 2005-2009.

Total de Revistas: 308

Total de Artículos: 929

Se identificaron un total de 3282 autores, de los cuales 42 investigadores publicaron más de siete artículos, otros 43 autores con más de cinco contribuciones, 243 entre tres y cuatro, 436 científicos con dos y 2518 investigadores publicaron 1 solo artículo. Un total de 42 autores constituyen los investigadores más productivos (1\% del total), este grupo se seleccionó para calcular los índices H, G, A y R (Tabla II). Además se determinó los índices H, G, A y R para aquellos autores que reciben mayor cantidad de citas, para verificar si los autores más productivos coinciden con los autores más citados. 


\begin{tabular}{|c|c|c|c|c|c|c|c|c|c|}
\hline Autor & $\begin{array}{c}\mathrm{N}^{\mathbf{0}} \\
\text { Artículos } \\
\end{array}$ & TACitados & $\begin{array}{c}\text { Índice } \\
\mathbf{H} \\
\end{array}$ & $\begin{array}{c}\text { Índice } \\
\mathbf{G}\end{array}$ & $\begin{array}{c}\text { Índice } \\
\mathbf{R}\end{array}$ & TCnH & $\begin{array}{c}\text { Índice } \\
\mathbf{A} \\
\end{array}$ & $\begin{array}{c}\text { Tcitas } \\
\text { R }\end{array}$ & $\begin{array}{c}\text { Prom } \\
\text { de } \\
\text { CXA } \\
\end{array}$ \\
\hline Pino, J. A. & 33 & 12 & 4 & 6 & 6 & 33 & 8 & 44 & 4 \\
\hline Arocha, Y. & 22 & 10 & 2 & 4 & 4 & 18 & 9 & 10 & 3 \\
\hline Marbot, R. & 21 & 9 & 2 & 5 & 5 & 25 & 12 & 9 & 4 \\
\hline Jones, P. & 16 & 10 & 2 & 4 & 4 & 18 & 9 & 10 & 3 \\
\hline Perez, O. & 14 & 10 & 3 & 4 & 4 & 16 & 5 & 25 & 3 \\
\hline Ly, J. & 12 & 3 & 2 & 3 & 3 & 9 & 5 & 10 & 3 \\
\hline Mas, R. & 12 & 8 & 2 & 2 & 2 & 15 & 3 & 15 & 2 \\
\hline Herrera, P. & 12 & 3 & 2 & 2 & 2 & 4 & 2 & 7 & 2 \\
\hline Payo, A. & 11 & 3 & 2 & 2 & 2 & 4 & 2 & 5 & 2 \\
\hline Stadler, M. & 11 & 5 & 2 & 2 & 2 & 6 & 3 & 10 & 2 \\
\hline Saikawa, M. & 10 & 5 & 2 & 2 & 2 & 5 & 3 & 22 & 9 \\
\hline Marti, M. P. & 10 & 5 & 2 & 4 & 4 & 17 & 9 & 20 & 4 \\
\hline $\begin{array}{l}\text { Castaneda Ruiz, } \\
\text { R. F. }\end{array}$ & 10 & 3 & 2 & 2 & 2 & 6 & 3 & 7 & 2 \\
\hline Lorenzo, J. C. & 10 & 3 & 1 & 1 & 1 & 2 & 2 & 4 & 1 \\
\hline Chao, D. & 10 & 3 & 2 & 2 & 2 & 4 & 2 & 5 & 2 \\
\hline Sierra, G. & 9 & 5 & 2 & 2 & 2 & 5 & 3 & 8 & 2 \\
\hline Bracho, G. & 9 & 6 & 3 & 4 & 4 & 16 & 5 & 19 & 3 \\
\hline Hernandez, M. & 9 & 5 & 2 & 4 & 4 & 17 & 9 & 23 & 5 \\
\hline Rodriguez, R. & 9 & 5 & 3 & 6 & 4 & 34 & 11 & 38 & 8 \\
\hline Almeida, R. & 8 & 5 & 2 & 4 & 4 & 16 & 8 & 20 & 4 \\
\hline Gil, D. & 8 & 5 & 2 & 2 & 3 & 7 & 4 & 10 & 2 \\
\hline Noa, M. & 8 & 4 & 2 & 2 & 2 & 4 & 2 & 6 & 2 \\
\hline Acevedo, R. & 8 & 3 & 2 & 2 & 3 & 7 & 4 & 8 & 3 \\
\hline Gonzalez, R. & 8 & 4 & 1 & 1 & 1 & 7 & 7 & 10 & 3 \\
\hline Minter, D. W. & 8 & 3 & 2 & 2 & 2 & 5 & 3 & 6 & 2 \\
\hline Guarro, J. & 8 & 3 & 2 & 2 & 2 & 5 & 3 & 6 & 2 \\
\hline Rastrelli, L. & 8 & 4 & 2 & 4 & 5 & 24 & 12 & 32 & 8 \\
\hline Gonzalez, C. & 8 & 1 & 1 & 1 & 1 & 1 & 1 & 3 & 3 \\
\hline Escalona, M. & 8 & 4 & 2 & 4 & 4 & 15 & 8 & 17 & 4 \\
\hline Pujol, M. & 8 & 7 & 2 & 3 & 3 & 8 & 4 & 14 & 2 \\
\hline $\begin{array}{l}\text { Borras-Hidalgo, } \\
\text { O. }\end{array}$ & 7 & 6 & 3 & 4 & 4 & 16 & 5 & 19 & 3 \\
\hline
\end{tabular}

Anales de Documentación, 2012, vol. 15, nº 1 


\begin{tabular}{|l|c|c|c|c|c|c|c|c|c|}
\hline Boa, E. & 7 & 0 & 0 & 0 & 0 & 0 & 0 & 0 & 0 \\
\hline Martin, C. & 7 & 4 & 2 & 3 & 3 & 11 & 6 & 16 & 3 \\
\hline Elias, A. & 7 & 0 & 0 & 0 & 0 & 0 & 0 & 0 & 0 \\
\hline Perez, Y. & 7 & 2 & 1 & 1 & 1 & 3 & 3 & 4 & 2 \\
\hline Mora, E. C. & 7 & 2 & 1 & 1 & 1 & 1 & 1 & 2 & 1 \\
\hline Lastre, M. & 7 & 5 & 2 & 2 & 3 & 7 & 4 & 10 & 2 \\
\hline Gonzalez, D. & 7 & 2 & 2 & 2 & 3 & 10 & 5 & 10 & 5 \\
\hline Gutierrez, A. & 7 & 2 & 2 & 2 & 2 & 5 & 3 & 5 & 3 \\
\hline Ortiz, M. & 7 & 2 & 1 & 1 & 1 & 1 & 1 & 2 & 1 \\
\hline Gusmao, L. F. P. & 7 & 2 & 1 & 1 & 1 & 1 & 1 & 2 & 1 \\
\hline Garcia, L. & 7 & 2 & 2 & 2 & 4 & 17 & 9 & 17 & 9 \\
\hline
\end{tabular}

Tabla II. Comportamiento de los indicadores calculados para 42 autores más productivos en el campo de las ciencias agropecuarias a partir de SCOPUS, 2005-2009.

No . de artículos: Número total de artículos publicados; TACitados: Número total de artículos citados; TCnH: total de citas en el núcleo H; Tcitas R: Total de citas recibidas; Prom de CXA: Promedio de citas por cada artículo.

Total de artículos: 929

Total de citas recibidas: 1245

Total de artículos citados: 346

Del total de autores que abordan las ciencias agropecuarias (3282 autores), 1422 científicos reciben citas; de ellos, 1159 (82\%) investigadores publicaron un solo artículo, por lo que su índice $\mathrm{H}$ es igual 1 . Del total de autores citados se seleccionaron 51 investigadores (4\%), los cuales recibieron más de cuatro citas; y se calculó para cada autor la batería de indicadores basados en los análisis de citas. De manera general, este grupo de autores publicó un total de 346 artículos y recibieron un total de 1245 citas.

Al comparar los resultados obtenidos en el grupo de autores con mayor actividad científica y los autores más citados, de manera general los autores más productivos coinciden con los autores más citados, con la excepción de los autores Boa, E. y Elías, A. que tienen publicados 7 artículos pero no han recibido citas.

\section{BATERÍA DE INDICADORES EVALUADOS}

El presente estudio permitió identificar que existe correlación entre los indicadores calculados a partir de los análisis de citas, específicamente se observó significativa correlación entre los índices H, G y R; así como también se demostró un crecimiento en los valores de los índices H, G y R a medida que aumenta la cantidad de publicaciones, además cuanto mayor es el número de citas que recibe cada autor aumentan los valores de 
los índices H, G y R. Importante señalar que los índices A, G y R son dependientes del total de citas en el núcleo H (Tabla III).

\begin{tabular}{|c|c|c|c|c|c|c|c|c|c|}
\hline & Prod. & TACitados & $\begin{array}{c}\text { Prom } \\
\text { de CXA }\end{array}$ & $\mathrm{TCnH}$ & TcitasR & $\begin{array}{c}\text { Índice } \\
\text { A }\end{array}$ & $\begin{array}{c}\text { Índice } \\
\mathrm{H}\end{array}$ & $\begin{array}{c}\text { Índice } \\
\text { G }\end{array}$ & $\begin{array}{c}\text { Índice } \\
\text { R }\end{array}$ \\
\hline Prod. & & 0,76 & 0,06 & 0,56 & 0,41 & 0,41 & 0,49 & 0,56 & 0,53 \\
\hline TACitados & 0,76 & & 0,17 & 0,68 & 0,58 & 0,57 & 0,68 & 0,74 & 0,72 \\
\hline Prom de CXA & 0,06 & 0,17 & & 0,59 & 0,69 & 0,67 & 0,43 & 0,52 & 0,60 \\
\hline $\mathrm{TCnH}$ & 0,56 & 0,68 & 0,59 & & 0,86 & 0,90 & 0,70 & 0,92 & 0,89 \\
\hline TcitasR & 0,41 & 0,58 & 0,69 & 0,86 & & 0,71 & 0,75 & 0,82 & 0,78 \\
\hline Índice A & 0,41 & 0,57 & 0,67 & 0,90 & 0,71 & & 0,51 & 0,83 & 0,85 \\
\hline Índice H & 0,49 & 0,68 & 0,43 & 0,70 & 0,75 & 0,51 & & 0,81 & 0,81 \\
\hline Índice G & 0,56 & 0,74 & 0,52 & 0,92 & 0,82 & 0,83 & 0,81 & & 0,92 \\
\hline Índice R & 0,53 & 0,72 & 0,60 & 0,89 & 0,78 & 0,85 & 0,81 & 0,92 & 1 \\
\hline
\end{tabular}

Tabla III. Matriz de correlación lineal de los principales indicadores en el campo de las ciencias agropecuarias cubanas a partir de SCOPUS.

Prod: Número total de artículos publicados; TACitados: Número total de artículos citados; TCnH: total de citas en el núcleo H; Tcitas R: Total de citas recibidas; Prom de CXA: Promedio de citas por cada artículo.

La función de distribución matemática que cumple la frecuencia de distribución de datos para cada uno de los índices H, G y R permite identificar, y a la vez visualizar, la frecuencia de asociación de las variables producción científica y los respectivos índices $\mathrm{H}$, G y R para los 42 autores más productivos en el sector agropecuario. Para el caso del Índice $\mathrm{H}$ constituye una variable discreta que cumple con una distribución normal, pero el mayor nivel de significación se logró cuando se ajusta esta variable a una distribución binomial, a pesar que los niveles de significación son muy bajos. En este comportamiento influyen determinados factores, como son el comportamiento de las citas de este grupo de investigadores, que pueden o no influir para que los valores de este índice cumplan con la Ley Gaussiana (Figura 2). En este sentido se verificó que, en la muestra estudiada, los autores más productivos tienen un índice H promedio igual a 2. 


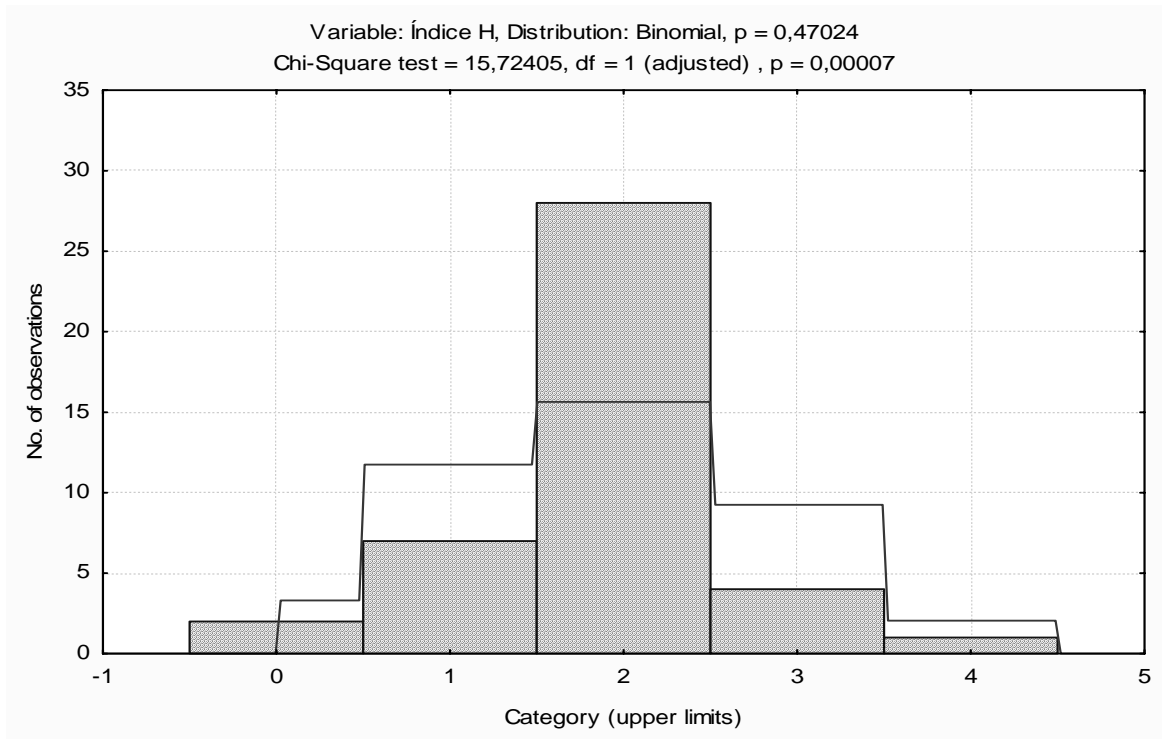

Figura 2. Distribución estadística en el índice $\mathrm{H}$ para 42 autores más productivos.

El índice G constituye una variable discreta, la cual se ajusta a una distribución normal pero con un bajo nivel de significación. Tanto el índice H como el índice G no siempre cumplen con una distribución estadística determinada, tema que se encuentra en estudio actualmente por especialistas en el tema. El promedio de citas recibidas para la muestra seleccionada es igual a 3 (Figura 3).

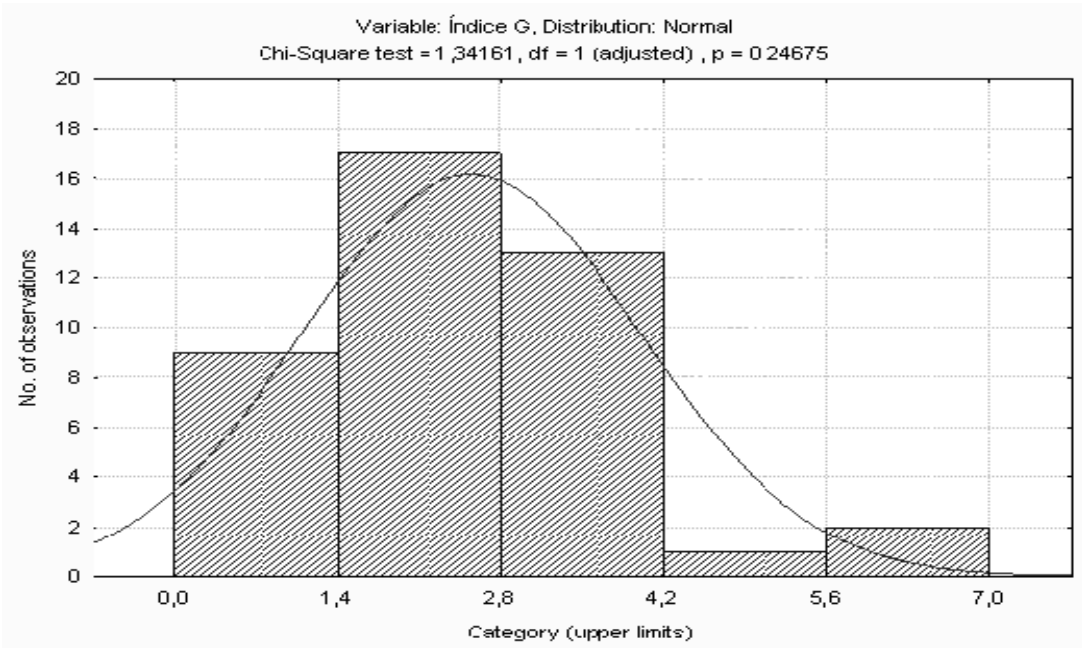

Figura 3. Distribución estadística en el índice G para 42 autores más productivos. 
Además, el índice $\mathrm{R}$ constituye una variable continua que, específicamente para el dominio de las ciencias agropecuarias, distribuye normal; este comportamiento permite corroborar que, para los 42 autores más productivos, el índice $\mathrm{R}$ promedio igual a 3 . El índice $\mathrm{R}$ constituye, de los tres índices, el índice más estático, característica estudiada previamente por Jin y colaboradores (Jin, Liang, Rousseau y Egghe, 2009, p. 239) (Figura 4).

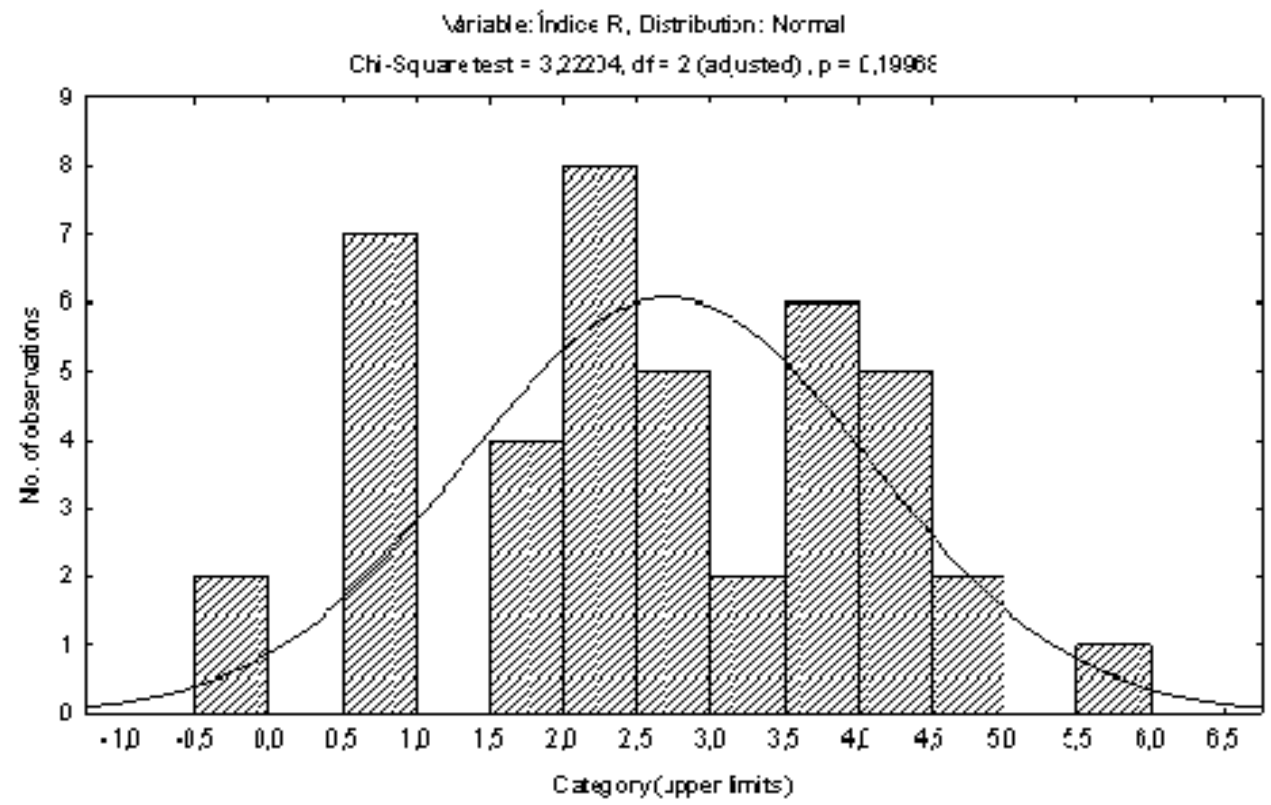

Figura 4. Distribución estadística en el índice $R$ para 42 autores más productivos.

\section{PRINCIPALES AUTORES EN EL SECTOR AGROPECUARIO CUBANO}

Los autores con mayor impacto en el campo de las ciencias agropecuarias cubanas se determinaron a partir de los siguientes indicadores calculados (Tablas IV, V, VI).

\begin{tabular}{|c|c|c|c|c|c|c|c|c|c|}
\hline Autor & $\begin{array}{c}\mathbf{N}^{0} \text { de } \\
\text { Artículos }\end{array}$ & TACitados & $\begin{array}{c}\text { Índice } \\
\mathbf{H} \\
\end{array}$ & $\begin{array}{c}\text { Índice } \\
\text { G } \\
\end{array}$ & $\begin{array}{c}\text { Índice } \\
\mathbf{R} \\
\end{array}$ & TCnH & $\begin{array}{c}\text { Índice } \\
\text { A } \\
\end{array}$ & $\begin{array}{c}\text { Tcitas } \\
\text { R } \\
\end{array}$ & $\begin{array}{c}\text { Prom de } \\
\text { CXA } \\
\end{array}$ \\
\hline Pino, J. A. & 33 & 12 & 4 & 6 & 6 & 33 & 8 & 44 & 4 \\
\hline Rodriguez, R. & 9 & 5 & 3 & 6 & 4 & 34 & 11 & 38 & 8 \\
\hline Perez, O. & 14 & 10 & 3 & 4 & 4 & 16 & 5 & 25 & 3 \\
\hline Bracho, G. & 9 & 6 & 3 & 4 & 4 & 16 & 5 & 19 & 3 \\
\hline Borras-Hidalgo, O. & 7 & 6 & 3 & 4 & 4 & 16 & 5 & 19 & 3 \\
\hline
\end{tabular}

Tabla IV. Autores más notables según índice $H$. 


\begin{tabular}{|c|c|c|c|c|c|c|c|c|c|}
\hline Autor & $\begin{array}{c}\mathbf{N}^{0} \text { de } \\
\text { Artículos }\end{array}$ & TACitados & $\begin{array}{c}\text { Índice } \\
\mathbf{H} \\
\end{array}$ & $\begin{array}{c}\text { Índice } \\
\text { G } \\
\end{array}$ & $\begin{array}{c}\text { Índice } \\
\mathbf{R} \\
\end{array}$ & TCnH & $\begin{array}{c}\text { Índice } \\
\text { A } \\
\end{array}$ & $\begin{array}{c}\text { Tcitas } \\
\text { R } \\
\end{array}$ & $\begin{array}{c}\text { Prom de } \\
\text { CXA } \\
\end{array}$ \\
\hline Pino, J. A. & 33 & 12 & 4 & 6 & 6 & 33 & 8 & 44 & 4 \\
\hline Rodriguez, R. & 9 & 5 & 3 & 6 & 4 & 34 & 11 & 38 & 8 \\
\hline Marbot, R. & 21 & 9 & 2 & 5 & 5 & 25 & 12 & 9 & 4 \\
\hline Perez, O. & 14 & 10 & 3 & 4 & 4 & 16 & 5 & 25 & 3 \\
\hline Bracho, G. & 9 & 6 & 3 & 4 & 4 & 16 & 5 & 19 & 3 \\
\hline Borras-Hidalgo, O. & 7 & 6 & 3 & 4 & 4 & 16 & 5 & 19 & 3 \\
\hline Arocha, Y. & 22 & 10 & 2 & 4 & 4 & 18 & 9 & 10 & 3 \\
\hline Jones, P. & 16 & 10 & 2 & 4 & 4 & 18 & 9 & 10 & 3 \\
\hline Marti, M. P. & 10 & 5 & 2 & 4 & 4 & 17 & 9 & 20 & 4 \\
\hline Hernandez, M. & 9 & 5 & 2 & 4 & 4 & 17 & 9 & 23 & 5 \\
\hline Almeida, R. & 8 & 5 & 2 & 4 & 4 & 16 & 8 & 20 & 4 \\
\hline Rastrelli, L. & 8 & 4 & 2 & 4 & 5 & 24 & 12 & 32 & 8 \\
\hline Escalona, M. & 8 & 4 & 2 & 4 & 4 & 15 & 8 & 17 & 4 \\
\hline
\end{tabular}

Tabla V. Autores más notables según índice G.

\begin{tabular}{|c|c|c|c|c|c|c|c|c|c|}
\hline Autor & $\begin{array}{c}\mathrm{N}^{0} \text { de } \\
\text { Artículos }\end{array}$ & TACitados & $\begin{array}{c}\text { Índice } \\
\text { H } \\
\end{array}$ & $\begin{array}{c}\text { Índice } \\
\text { G }\end{array}$ & $\begin{array}{c}\text { Índice } \\
\mathbf{R} \\
\end{array}$ & TCnH & $\begin{array}{c}\text { Índice } \\
\text { A } \\
\end{array}$ & $\begin{array}{c}\text { Tcitas } \\
\mathbf{R} \\
\end{array}$ & $\begin{array}{c}\text { Prom de } \\
\text { CXA }\end{array}$ \\
\hline Pino, J. A. & 33 & 12 & 4 & 6 & 6 & 33 & 8 & 44 & 4 \\
\hline Marbot, R. & 21 & 9 & 2 & 5 & 5 & 25 & 12 & 9 & 4 \\
\hline Rastrelli, L. & 8 & 4 & 2 & 4 & 5 & 24 & 12 & 32 & 8 \\
\hline Arocha, Y. & 22 & 10 & 2 & 4 & 4 & 18 & 9 & 10 & 3 \\
\hline Jones, P. & 16 & 10 & 2 & 4 & 4 & 18 & 9 & 10 & 3 \\
\hline Marti, M. P. & 10 & 5 & 2 & 4 & 4 & 17 & 9 & 20 & 4 \\
\hline Hernandez, M. & 9 & 5 & 2 & 4 & 4 & 17 & 9 & 23 & 5 \\
\hline Garcia, L. & 7 & 2 & 2 & 2 & 4 & 17 & 9 & 17 & 9 \\
\hline Perez, O. & 14 & 10 & 3 & 4 & 4 & 16 & 5 & 25 & 3 \\
\hline Bracho, G. & 9 & 6 & 3 & 4 & 4 & 16 & 5 & 19 & 3 \\
\hline Borras-Hidalgo, O. & 7 & 6 & 3 & 4 & 4 & 16 & 5 & 19 & 3 \\
\hline Almeida, R. & 8 & 5 & 2 & 4 & 4 & 16 & 8 & 20 & 4 \\
\hline Escalona, M. & 8 & 4 & 2 & 4 & 4 & 15 & 8 & 17 & 4 \\
\hline
\end{tabular}

Tabla VI. Autores más notables según índice R.

El análisis de los tres indicadores permitió identificar de manera general un grupo de autores que coincidentemente se destacan en el campo de las ciencias agropecuarias cubanas según los índices H, G, R y el total de citas que reciben los artículos científicos. Se puede mencionar el Dr. Jorge A. Pino Alea, investigador del Instituto de Investigaciones para la Industria Alimenticia Ciudad de la Habana (Cuba): la mayoría de sus contribuciones abordan temáticas enfocadas hacia el desarrollo de la química y 
bioquímica de frutas y vegetales, además es especialista en aromas y aceites esenciales; MsC. Rafael Rodríguez Hernández, miembro del grupo de investigación de Fisiología digestiva del Instituto de Ciencia Animal; y el Dr. Oliver Pérez, investigador procedente del Instituto Finlay. Además, se destacan autores como el Dr. Orlando Borrás Hidalgo, Jefe de Laboratorio de Genómica Funcional de Plantas, del Centro de Ingeniería Genética y Biotecnología; G. Bracho, investigador del Departamento de Inmunología, Instituto Finlay; Magalis Hernández, del Centro de Bioplantas de Ciego de Ávila; M. P. Martín, profesor de la Universidad Pública de Tarragona; y R. Almeida, investigador del Instituto Nacional de Investigaciones de la Caña de Azúcar.

Además, se puede observar en las tablas IV, V y VI el caso de "Rafael Rodríguez Hernández”, investigador con una producción moderada (9 publicaciones) pero que cuenta con un promedio de 8 citas por documento. Al comparar este investigador con "Jorge Pino" se puede observar como, con menos de un tercio de la producción de Pino, Rodríguez consigue casi el mismo número de citas (44 vs. 38); es decir, en base a estos indicadores, Rodríguez puede ser considerado como un investigador más "eficiente" que Pino; sin embargo en base al índice H, Pino seria considerado “mejor” que Rodríguez.

\section{DISCUSIÓN / CONCLUSIONES}

Los investigadores en el sector agropecuario cubano no solo publican sus resultados en la revista Cuban Journal of Agricultural Science, sino que, también envían sus trabajos a revistas extranjeras, por lo que se denota como los científicos cubanos en el dominio de las ciencias agropecuarias logran romper las barreras que imponen modelos de comunicación científica de primer mundo. También se debe mencionar que hay un gran porciento de autores que publican ocasionalmente y en este sentido es necesario fomentar los trabajos con alto rigor científico, para así aumentar la producción científica y por consiguiente el desarrollo de la ciencia cubana.

Las publicaciones con altos valores según el Scimago Journal Rank (SJR) constituyen las revistas donde los autores con mayor índice $\mathrm{H}$ publican sus resultados científicos. En la actualidad, las publicaciones científicas que se encuentran indizadas en la base de datos Scopus exigen artículos con mayor calidad y originalidad. De esta manera, los autores con prestigio científico dentro una rama del conocimiento procuran publicar sus resultados en revistas que sean visibles dentro del gremio científico en el cual trabajan y al mismo tiempo tienen la oportunidad de encontrar y recibir reconocimiento a través de las citas.

En la muestra de autores estudiados, los valores que ofrece el cálculo del índice $\mathrm{H}$ fundamentalmente favorece a los investigadores consolidados dentro de la comunidad científica, los cuales presentan altos niveles de producción y citas recibidas, mientras que autores menos productivos se ven afectados cuando son evaluados en base a estos indicadores, fenómeno que, se aborda en la literatura (Egghe, 2010, p. 6), (Alonso; Cabrerizo; Herrera-Viedma y Herrera, 2010 p. 392), (Liu y Rousseau, 2009, p. 237), (Costas y Bordons, 2007, p. 196) como una desventaja del índice H. Por tal motivo el 
estudio centra su atención en el análisis de 42 investigadores que, básicamente son los que tienen más producción, lo cual representa una población de análisis relativamente pequeña y sesgada hacia los mayores productores.

Los autores con mayor producción científica recibieron los mejores valores en cuanto al total de citas recibidas se refiere. Este grupo de autores alcanzaron, además, los mejores promedios de citas por trabajo publicado y lograron los valores más altos en el cálculo del índice H. En este sentido se puede afirmar que, de manera general, los autores con altos niveles de producción reciben mayor número de citas. Para evaluar actualmente el desempeño científico de un autor es necesario realizar un análisis cuantitativo y cualitativo del mismo. Por ello construir indicadores de producción e impacto permite obtener una calificación del estado científico del autor. En este sentido, el índice H en combinación con otros indicadores bibliométricos es empleado para evaluar la producción científica y el impacto de la investigación.

Se confirma una vez más la alta correlación entre el índice H, G y R; así como su relación con el nivel de producción y la cantidad de citas que recibe el autor; porque a medida que aumenta el número de contribuciones por cada autor, aumenta el comportamiento de índices evaluados, así como también, mayores serán los valores de los índices H, G y R cuando los investigadores reciben mayor cantidad de citas. En este sentido, estudios realizados por Bornmann y otros; (Bornman; Mutz y Hans-Dieter, 2009, p. 1288) (Costas y Bordons, 2008, p. 77); demuestran, a través de métodos estadísticos, la alta intercorrelación existente entre el índice $\mathrm{H}$ y el índice $\mathrm{G}$ utilizando el núcleo de artículos más productivos, para obtener una dimensión cuantitativa del fenómeno que se pretende evaluar. Por otro lado, el índice A y el índice R presentan alta relación con el total de citas recibidas, en aras de validar cualitativamente la trayectoria académica de un científico.

Al analizar las distribuciones estadísticas de los índices $\mathrm{H}, \mathrm{G}$ y R de los autores en el sector agropecuario cubano, en relación con la cantidad de artículos publicados, se determinaron bajos niveles de significación, característica que denota un sesgo en el comportamiento estadístico de estos índices en proporción con la producción científica, fenómeno relacionado con la distribución de frecuencias de la Ley de Lotka (Egghe, 2007, p. 2). Se corrobora, una vez más, el sesgo hacia aquellos científicos con altos niveles de producción, los cuales constituyen los investigadores más visibles en el sector agropecuario cubano.

La aplicación de estos índices en nuestro país, desde una perspectiva evaluadora a nivel micro, constituye una vía para las universidades y centros de investigación para seleccionar científicos y profesores activos en la investigación, sin dejar a la deriva criterios establecidos en el Decreto Ley 104 tales como, la categoría científica del investigador, informes y trabajos presentados en eventos, publicaciones, conocimientos de otros idiomas, superación autodidacta, así como, cumplir con las funciones específicas de la actividad científica. 


\section{BIBLIOGRAFÍA}

ALONSO, S.; CABRERIZO, F.J.; HERRERA-VIEDMA, E. y HERRERA, F. H G-index: A new index to characterize the scientific output of researchers based on the h- and gindices. Scientometrics, 2010, vol. 82, n 2, p. 391-400.

ARENCIBIA-JORGE, R.; BARRIOS-ALMAGUER, I.; FERNÁNDEZ-HERNÁNDEZ, S. y CARVAJAL-ESPINO, R. Applying successive $\mathrm{H}$ indices in the institutional evaluation. Journal for the American Society of Information Science and Technology, 2008, vol. 59, no 1, p. 155-157.

ARENCIBIA-JORGE, R. y CARVAJAL-ESPINO, R. Los índices H, G y R: su uso para identificar autores líderes en el área de la comunicación durante el período 2001-2006. Acimed, 2008, vol. 17, $n^{\circ}$ 4, p. 1-9.

BALL, P. Achievement index climbs the ranks. Nature, 2007, vol. 448, ${ }^{\circ} 737$.

BORNMANN, L. y HANS-DIETER, D. The state of $h$ index research. Is the $h$ index the ideal way to measure research performance? European Molecular Biology Organization, 2009, vol. 10, $\mathrm{n}^{\circ}$ 1, p. 2-6.

BORNMANN, L.; MUTZ, R. y HANS-DIETER, D. Do We Need the h Index and Its Variants in Addition to Standard Bibliometric Measures? Journal of the American Society for Information Science and Technology, 2009, vol. 60, nº 6, p. 1286-1289.

BRAUN, T.; GLÄNZEL, W. y SCHUBERT, A. A Hirsch-type index for journals. Scientometrics, 2006, vol. 69, n ${ }^{\circ}$ 1, p. 169-173.

COSTAS, R. y BORDONS, M. The h-index: Advantages, limitations and its relation with other bibliometric indicators at the micro level. Journal of Informetrics, 2007, vol. 1, $\mathrm{n}^{\circ}$ 3, p. 193-203.

COSTAS, R. y BORDONS, M. Is g-index better than h-index? An exploratory study at the individual level. Scientometrics, 2008, vol. 77, n 2, p. 267-288.

DORTA-CONTRERAS, A.J.; ARENCIBIA-JORGE, R.; MARTÍ-LAHERA, Y. y ARAUJO-RUIZ, J.A. Productividad y visibilidad de los neurocientíficos cubanos: estudio bibliométrico del período 2001-2005. Revista de Neurología, 2008, vol. 47, $\mathrm{n}^{\circ}$ 7, p. 355-360.

EGGHE, L. Theory and practise of the g-index. Scientometrics, 2006, vol. 69, $\mathrm{n}^{\mathrm{o}} 1$, p. 131-152.

EGGHE, L. Distributions of the h-index and the g-index. Paper presented at the Proceedings of the 11th International Society, 2007.

EGGHE, L. The Hirsch-Index and Related Impact Measures. Annual Review of Information Science of Technology, 2010, vol. 44, p. 3-9.

HIRSCH, J.E. An index to quantify an individual's scientific research output. PNAS, 2005, vol. 102, no 46, p. 16569-16572.

JIN, B.; LIANG, L.; ROUSSEAU, R. y EGGHE, L. The R- and AR-indices: Complementing the h-index. Scientometrics, 2009, vol. 79, n 2 , p. 235-248.

LIU, Y. y ROUSSEAU, R. Properties of Hirsch-type indices: The case of library classification categories. Scientometrics, 2009, vol. 79, nº 2, p. 235-248.

RUIZ-PEREZ, R.; DELGADO LÓPEZ-CÓZAR, E. y JIMÉNEZ-CONTRERAS, E. Principios y criterios utilizados en España por la Comisión Nacional Evaluadora de la 
Actividad Investigadora (CNEAI) para la valoración de las publicaciones científicas: 1989-2009. Psicothema, 2010, vol. 22, nº 4, p. 898-908.

SCIMAGO, G. El índice h de Hirsch: aportaciones a un debate. El Profesional de la Información, 2006, vol. 15, nº 4, p. 304-306. 